\title{
Estimation of the Amplitude of Resonance in the General Standard Map
}

\author{
Arturo Olvera
}

\section{CONTENTS}

1. Introduction

2. Resonant Normal Forms

3. Rules to Determine the Resonant Normal Form

4. Numerical computation

5. Estimate of $\Delta_{\mathrm{p} / \mathrm{q}}$ for Values of $\varepsilon$ of the Order of 1

6. Collapse of Resonance

7. An Example of Analytical Perturbation

8. Conclusion

Appendix A: Hamiltonian Flows and Symplectic Twist Maps Appendix B: Amplitude of Resonance in the Generic Case Appendix C: Alternative Method to Find a Resonant Normal Form

Acknowledgements

Bibliography
This paper formulates some conjectures about the amplitude of resonance in the General Standard Map. The main idea is to expand the periodic perturbation function in Fourier series. Given any rational rotation number, we choose a finite number of harmonics in the Fourier expansion and we compute the amplitude of resonance of the reduced perturbation function of the map, using a suitable normal form around the resonance, which is valid for asymptotically small values of the perturbation parameter. For this map, we obtain a relation between the amplitude of resonance and the perturbation parameter: the amplitude is proportional to a rational power of the parameter, and so can be represented as a straight line on a log-log graph. The convex hull of these straight lines gives a lower bound for the amplitude of resonance, valid even when the perturbation parameter is of the order of 1 . We find that some perturbation functions give rise a phenomenon that we call collapse of resonance; this means that the amplitude of resonance goes to zero for some value of the perturbation parameter. We find an empirical procedure to estimate this value of the parameter related to the collapse of resonance.

\section{INTRODUCTION}

The study of stability and the chaotic behavior of Hamiltonian systems with two degrees of freedom is an important problem in classical mechanics and dynamical systems. The problem of finding the threshold of stability has been studied by many authors; outstanding contributions were made by Chirikov and Escande in the early 1970s.

It is well known that a Hamiltonian flow can be reduced to a two-dimensional map using a Poincaré section; in that form the dynamics of the Hamiltonian flow can be studied in terms of the stability problem of the two-dimensional map. To find the regions of stability and the threshold of chaotic behavior, we can use the Chirikov [1979] overlap method. The idea of this method is to obtain the shape of the 
invariant manifolds of the hyperbolic periodic orbits of our map; a pictorial description of these manifolds looks like a chain of pendulum separatrices (see Figure 1). It is common to call resonances the structures defined by the hyperbolic periodic points and their invariant manifolds (or separatrices). The resonances are denoted by the rotation number of the corresponding periodic orbit. Chirikov studied the interaction of two resonances: when the separatrices of these two resonances overlap, we can expect to find chaotic behavior around the resonances. A similar idea can be found in [Escande 1982; Olvera and Simó 1987].

One important aspect of the overlap method is the necessity to estimate the amplitude of the resonances. This amplitude corresponds to the maximum distance of the separatrices (in Figure 1, the maximum distance is given in the vertical direction). We denote this amplitude by $\Delta_{p / q}$, where the subscript $p / q$ is the rotation number of the periodic orbit, and therefore a rational number.

In many cases, the two-dimensional map can be described as the sum of an integrable twist map and a small perturbation. The amplitude of the perturbation is driven by one real parameter. The goal of this paper is to obtain a simple relation between the perturbation parameter and the amplitude of resonance for any rational rotation number.

In this work we choose a particular (parametrized) twist map, known as the Standard Map. This twodimensional map is important in the study of Hamiltonian systems with two degrees of freedom; Chirikov [1979] and Lichtenberg [1983] describe many examples of how we can reduce the Hamiltonian flow to a Standard Map. In this case, when we are interested in the dynamics around any fixed point (or periodic orbit) of any Hamiltonian system with two degrees of freedom, it is easy to find a corresponding twist map representing the dynamics around the fixed point. This procedure is described in Appendix A.

We now define the General Standard Map and study its resonances and their relation with the perturbation parameter. This map is defined by

$$
\rho_{i+1}=\rho_{i}-\varepsilon V\left(\varphi_{i}, \varepsilon\right), \quad \varphi_{i+1}=\varphi_{i}+\rho_{i+1} .
$$

The radial variable $\rho_{i} \in \mathbb{R}$ and the angular variable $\varphi \in S^{1}$. The perturbation function $V(\varphi, \varepsilon)$ is

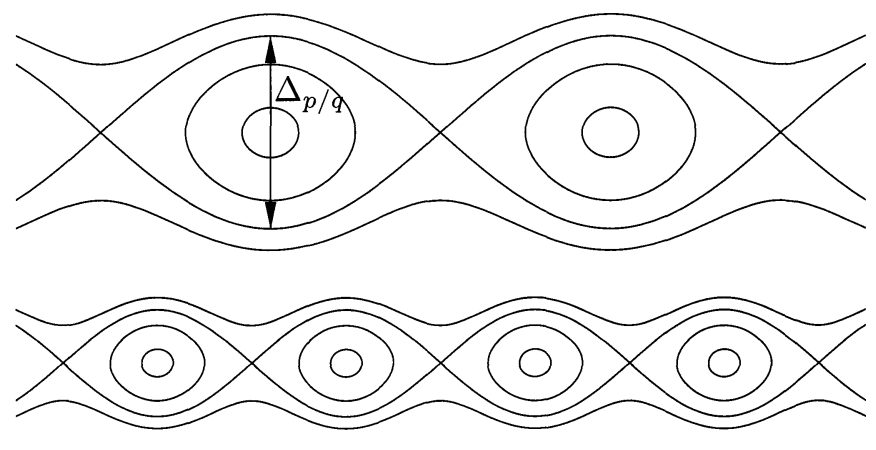

FIGURE 1. Phase space of two resonances.

analytic and periodic with respect to the angular variable, that is, $V(\varphi+1, \varepsilon)=V(\varphi, \varepsilon)$. The perturbation parameter $\varepsilon$ is a nonnegative real number. For $\varepsilon=0$, the map (1-1) reduces to an integrable twist map and the phase space $\left(\mathbb{R} \times S^{1}\right)$ is completely foliated by invariant circles. For any value of the radial variable $\rho$, there exists an invariant circle with rotation number $\rho$.

For a small perturbation, $0<\varepsilon \ll 1$, we can use the Birkhoff twist theorem to show that the set of invariant circles with rational rotation number disappears (in the generic sense), and it is transformed into an even number of periodic orbits with the same rational rotation number. The linear stability of these orbits is elliptic and hyperbolic. For asymptotically small values of the parameter, it is possible to find the amplitude of any resonance (in the generic case) of the corresponding hyperbolic orbit; this amplitude is of the form $\Delta_{p / q} \sim \varepsilon^{1 / 2}$. Appendix $\mathrm{B}$ shows the derivation of this well-known relation.

The main contribution of this paper is to find the way in which the amplitude of resonance $\Delta_{p / q}$ depends on the perturbation parameter $\varepsilon$ when $\varepsilon$ is not asymptotically small; we can describe $\Delta_{p / q}$ all the way up to $\varepsilon$ of the order of 1 . We also show that the behavior of the amplitude of resonance is not homogeneous over the whole range of $\varepsilon$. More precisely, we can write $\Delta_{p / q}=A_{i} \varepsilon^{n_{i}}$ for $\varepsilon \in\left(\varepsilon_{i}, \varepsilon_{i+1}\right)$, with different rational exponents $n_{i}$ in different intervals, and we can determine the borderline values $\varepsilon_{i}$ where the rate of growth of the amplitude of resonance changes.

Our procedure to find the amplitude of resonance $\Delta_{p / q}$ for any value of the perturbation parameter $\varepsilon$ has four steps. 
1. Get the Fourier expansion of the perturbation function $V(\varphi, \varepsilon)$ and define a new perturbation function $V_{N}(\varphi, \varepsilon)$ by selecting some of the the first $N$ harmonics of $V(\varphi, \varepsilon)$ (where $N \geq q$ ).

2. Use $V_{N}(\varphi, \varepsilon)$ as the perturbation function of the Standard Map (1-1). Compute an appropriate normal form via the Poincaré-Lindstedt method. Use this normal form to determine the dynamics close to the periodic orbit which looks like the periodic orbits of the pendulum equation; this makes it easy to find the amplitude of the separatrices of this pendulum equation. The normal form obtained is only valid for asymptotically small values of $\varepsilon$.

3. Repeat the first two steps several times, changing the set of selected harmonics to form the function $V_{N}(\varphi, \varepsilon)$.

4. Find that the behavior of $\Delta_{p / q}$, for a large value of $\varepsilon$, as the direct sum of the amplitudes of resonance obtained in the previous steps.

We conclude that only a few harmonics of the Fourier expansion of $V(\varphi, \varepsilon)$ are responsible for the behavior of $\Delta_{p / q}$ in each interval of the domain of $\varepsilon$. Note that we can predict the size of $\Delta_{p / q}$ for a large value of $\varepsilon$ using only asymptotic information obtained from the normal forms computed in the Standard Map using different perturbation functions $V_{N}(\varphi, \varepsilon)$.

Section 2 of the paper describes the method of obtaining the normal form for perturbation functions defined as a trigonometric polynomial of $\varphi$. This normal form only depends on the rotation number and the set of harmonics that form the perturbation function. Section 3 shows how we can get this kind of normal form using some information on the linear stability of the hyperbolic periodic orbit. In order to judge the accuracy of our estimate of $\Delta_{p / q}$, we develop an alternative, numerical, method to compute it; this is done in Section 4. We give examples in Section 5 comparing the amplitude of resonance obtained by the numerical and analytic methods. Finally, Section 6 describes an interesting phenomenon: for some values of $\varepsilon$ the amplitude of resonance goes to zero. We call this the collapse of resonance.

\section{RESONANT NORMAL FORMS}

In this section we work out a procedure to obtain a map that displays in a simple form the dynamics around a specific periodic orbit. The idea is to perform a set of coordinate transformations in order to obtain a new map that must be closer to an integrable map (in the neighborhood of the given rotation number) than the original one. We show that we need only perform a finite number of these transformations if we set the rotation number as a rational number $p / q$ with $(p, q)=1$. The final map can be related to a simple Hamiltonian flow. The dynamics of the Hamiltonian system is close to the final map up to some order of the perturbation parameter $\varepsilon$. Our procedure is similar to the Lindstedt series method, a standard procedure to solve nonlinear differential equations using asymptotic methods in Celestial Mechanics. In order to perform symplectic transformations we must rewrite our map. We take the two difference equations of $(2-3)$ and rewrite it as one second order difference equation, which we call the Lagrangian representation of the Standard Map.

Consider the map (1-1), where the perturbation function $V(\varphi, \varepsilon)$ is an analytic function with null average and periodic: $V(\varphi+1, \varepsilon)=V(\varphi, \varepsilon)$. A general expression for this function is given by the Fourier expansion of the angular variable, the coefficient of each Fourier term being a power series in $\varepsilon$ :

$$
V(\varphi, \varepsilon)=\sum_{s=1}^{\infty} \sum_{j=-\infty}^{\infty} C_{s, j} \varepsilon^{s} e^{2 \pi i j \varphi}
$$

We can obtain a good approximation of the perturbation function taking only the first $N$ harmonic terms of the preceding equation, where $N \leq q$. Each harmonic is multiplied by the leading term of the corresponding power series of the perturbation parameter. Our approximation is then given by

$V(\varphi, \varepsilon)=\sum_{j=1}^{N} \varepsilon^{a_{j}}\left(c_{j} e^{2 \pi i w_{j} \varphi}+\hat{c}_{j} e^{-2 \pi i w_{j} \varphi}\right)+O\left(\varepsilon^{S_{N}}\right)$,

where the $a_{i}$ are positive integers, the frequencies $w_{i}$ are positive integers such that $w_{i}<w_{i+1}$ and $c_{i}, \hat{c}_{i}$ are complex numbers not depending on $\varepsilon$. The remaining term $O\left(\varepsilon^{S_{N}}\right)$, where $S_{N}>\max _{j=1, \ldots, N}\left\{a_{j}\right\}$, does not contain harmonics of order less than $w_{N}$. 
We assume that the magnitude of the coefficients of the harmonic terms that belong to $O\left(\varepsilon^{S_{N}}\right)$ is smaller than

$$
\min _{j=1, \ldots, N}\left\{c_{j}, \hat{c}_{j}\right\} .
$$

If $V(\varphi, \varepsilon)$ is analytic, we can see that these coefficients $c_{j}$ and $\hat{c}_{j}$ are proportional to $\gamma^{j}$, where $\gamma$ is a constant such that $|\gamma|<1$ and $a_{j}=0$.

In order to find the resonant normal form we must take the lift of our map (1-1). For this map, the phase space is now $\mathbb{R}^{2}$. We can write the lift in the form

$$
y_{i+1}=y_{i}+\varepsilon V\left(x_{i}, \varepsilon\right), \quad x_{i+1}=x_{i}+y_{i+1},
$$

where $\left(x_{i}, y_{i}\right) \in \mathbb{R}^{2}$ and $i \in \mathbb{Z}$. This map is a set of two difference equations of first order; we can rewrite it as a single difference equation of second order, and this is the Lagrangian form of (1-1):

$$
x_{i+1}-2 x_{i}+x_{i-1}=\varepsilon V\left(x_{i}, \varepsilon\right) .
$$

We want to find a coordinate transformation that conjugates, as far as possible, the dynamics of equation $(2-3)$ to the dynamics of an integrable twist map in the neighborhood of a given rotation number $p / q$. The dynamics are given as a uniform rotation:

$$
\theta_{i+1}=\theta_{i}+\frac{p}{q}
$$

where $\theta_{i} \in \mathbb{R}$.

Let the function $g(\theta, \varepsilon)$ define the conjugation between the coordinate $x$ and the coordinate $\theta$ :

$$
x_{i}=\theta_{i}+g\left(\theta_{i}, \varepsilon\right) .
$$

The function $g(\theta, \varepsilon)$ is periodic and we can expand it as a power series in $\varepsilon$ where the coefficients are periodic functions of $\theta$ and are given by Fourier series:

$$
g(\theta, \varepsilon)=\sum_{j=0}^{\infty} \varepsilon^{j} \sum_{k \in \mathbb{Z}} g_{j, k} e^{2 \pi i k \theta} .
$$

Substituting this and (2-5) into (2-3), and taking into account that the dynamic of $\theta$ is a uniform rotation (2-4), we obtain

$$
\begin{array}{r}
\theta_{i+1}-2 \theta_{i}+\theta_{i-1}+\sum_{j=0}^{\infty} \varepsilon^{j} \sum_{k \in \mathbb{Z}} g_{j, k} e^{2 \pi i k \theta} 2\left(\cos \frac{2 \pi j p}{q}+1\right) \\
=\sum_{l=1}^{N} \varepsilon^{a_{l}}\left(c_{l}^{+} e^{2 \pi i w_{l}\left(\theta_{i}+g(\theta, \varepsilon)\right)}+c_{l}^{-} e^{-2 \pi i w_{l}\left(\theta_{i}+g(\theta, \varepsilon)\right)}\right) .
\end{array}
$$

The right-hand side is an exponential function whose arguments include the function $g(\theta, \varepsilon)$, so we can expand the exponential function as a power series of $g(\theta, \varepsilon)$ :

$$
\begin{aligned}
& e^{ \pm 2 \pi i w_{l}(\theta+g(\theta, \varepsilon))} \\
& =e^{ \pm 2 \pi i w_{l} \theta}\left(1+\sum_{s=1}^{\infty} \frac{1}{s !}\left( \pm \sum_{j=0}^{\infty} \varepsilon^{j} \sum_{k \in \mathbb{Z}} g_{j, k} e^{2 \pi i k \theta}\right)^{s}\right) .
\end{aligned}
$$

Substituting this into the preceding equation and considering that $\theta_{i+1}-2 \theta_{i}+\theta_{i-1}$ must be 0 (because the dynamic of $\theta$ is a uniform rotation), we get

$$
\begin{aligned}
\sum_{j=0}^{\infty} \varepsilon^{j} \sum_{k \in \mathbb{Z}} g_{j, k} e^{2 \pi i k \theta} 2\left(\cos \frac{2 \pi j p}{q}-1\right) \\
=\sum_{l=1}^{N} \varepsilon^{a_{i}}\left(c _ { i } ^ { \pm } e ^ { \pm 2 \pi i w _ { l } \theta } \left(1+\sum_{s=1}^{\infty} \frac{1}{s !}\right.\right. \\
\left.\left.\times\left( \pm \sum_{j=0}^{\infty} \varepsilon^{j} \sum_{k \in \mathbb{Z}} g_{j, k} e^{2 \pi i k \theta}\right)^{s}\right)\right) .
\end{aligned}
$$

To determine the values of the coefficients $g_{j, k}$ we must solve (2-6). For any positive integer $m$ we can collect all the terms of order $O\left(\varepsilon^{m}\right)$; these terms depend on the coefficients $g_{j, k}$, where $j=0, \ldots, m-1$ and $k$ is a finite subset of $\mathbb{Z}$. The coefficient $g_{m, k}$ appears in a linear form in the left side of equation (2-6). Then we have an infinite number of homological equations, each related to the same order of the perturbation parameter, $\varepsilon^{m}$, and a specific harmonic term $e^{2 \pi i k \theta}$. Such a homological equation can be expressed as

$$
\begin{aligned}
& 2 g_{m, k}\left(\cos \frac{2 \pi k p}{q}-1\right) \\
& \quad=G_{m, k}\left(g_{0, k^{\prime}}, g_{1, k^{\prime \prime}}, \ldots, g_{m-1, k^{(m)}}\right),
\end{aligned}
$$

where $k, k^{\prime}, \ldots, k^{(m)} \in \mathbb{Z}$. We can solve $(2-7)$ because its right-hand side depends only on the coefficients $g_{i, k}$ that we have already computed. Note that for fixed order of $\varepsilon$, the right-hand side of (2-6) has a finite number of harmonic terms, so that for any order of $\varepsilon$ we must solve a finite number of homological equations.

Two conditions must be satisfied if we want to solve the homological equations (2-7):

1. For any order of $\varepsilon$, the right side of equation (2-6) must have null average. 
2. The term $\cos (2 \pi k p / q)-1$ must be nonzero.

The first condition means that for any value of $m$ in $(2-7)$ the term $G_{m, 0}\left(g_{0, k}, \ldots, g_{m-1, k^{(m)}}\right)$, which corresponds to a harmonic term of frequency zero, must vanish in order to have null average. As a consequence, the coefficients $g_{m, 0}$ are indeterminate and we can fix any value for them. We can use these free parameters to ensure that $G_{m, 0}\left(g_{0, k}, \ldots, g_{m-1, k^{(m)}}\right)$ vanishes.

The second condition is the nonresonance condition. If the value of $k$ is $\pm q$ then $\cos (2 \pi k p / q)-1=0$. In this case there are no solutions of the homological equation $(2-7)$. Therefore we cannot continue the procedure to solve the homological equation for the next order of $\varepsilon$. This condition defines the resonant normal form. There are some cases where $G_{m, \pm q}=0$; then the homological equation is trivial and we can continue our procedure until we can reach the next nonresonance condition when $k=2 q$.

We can only solve a finite number of homological equations $(2-7)$ because there exists an integer $\bar{m}$ such that the nonresonant condition is not satisfied. For this value of $\bar{m} \in \mathbb{N}$ there are terms of order $O\left(\varepsilon^{\bar{m}}\right)$ that contain harmonic terms with frequency $\pm q$ (and $G_{\bar{m}, \pm q}\left(g_{j, k}\right) \neq 0$ in general). As a consequence, it is impossible to conjugate the dynamics of our map (2-3) to the dynamics of a uniform rotation in the neighborhood of the rotation number $p / q$. Therefore $\theta_{i+1}-2 \theta_{i}+\theta_{i-1}=O\left(\varepsilon^{\bar{m}}\right) \neq 0$ for $i \in \mathbb{Z}$. Hence, we say that this equation is a resonant normal form and the dynamics of the coordinate $\theta$ are then described in the form

$\theta_{i+1}-2 \theta_{i}+\theta_{i-1}=\varepsilon^{\bar{m}} G_{\bar{m}, \pm q}\left(g_{j, k}\right) e^{ \pm 2 \pi i q \theta_{i}}+O\left(\varepsilon^{\bar{m}+1}\right)$.

We can shift the origin of the coordinate $\theta$ and we can rewrite the preceding equation in the form

$$
\theta_{i+1}-2 \theta_{i}+\theta_{i-1}=\varepsilon^{\bar{m}} A_{p / q} \sin \left(2 \pi q \theta_{i}\right)+O\left(\varepsilon^{\bar{m}+1}\right)
$$

We divide equation (2-8) by $\varepsilon^{\bar{m}}$, then we can interpret the left side as the approximation of a second derivative of the variable $\theta$ respect to time, where $\Delta t=\sqrt{\varepsilon^{\bar{m}}}$. The related differential equation is the pendulum equation:

$$
\frac{d^{2} \theta}{d t^{2}}=A_{p / q} \sin (2 \pi q \theta)
$$

Figure 2 shows the phase space of this equation. The phase space of our resonant normal form (2-8) is similar to Figure 2 to order $\varepsilon^{\bar{m}+1}$. We can see that there are $2 q$ periodic points which correspond to the image of two periodic orbits, the first one has elliptic linear stability and the second one is hyperbolic.

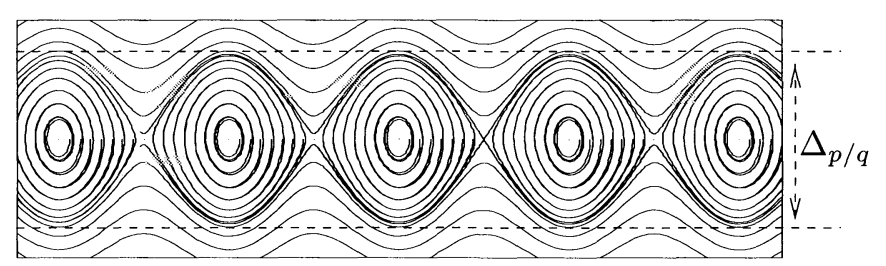

FIGURE 2. Phase space of the $q$-pendulum equation.

The maximum amplitude of the separatrices of equation (2-9) is $\Delta=4 \sqrt{A_{p / q} /(2 \pi q)}=4 \lambda /(2 \pi q)$, where $\lambda$ is the eigenvalue of the hyperbolic point. The corresponding value of the amplitude of resonance related to the resonant normal form $(2-8)$ is then

$$
\Delta_{p / q}=\varepsilon^{\bar{m} / 2} \sqrt{\frac{A_{p / q}}{2 \pi q}}+O\left(\varepsilon^{(\bar{m}+1) / 2}\right),
$$

and the eigenvalues of the hyperbolic points are

$$
\lambda_{p / q}=1 \pm \varepsilon^{\bar{m} / 2} \sqrt{2 \pi q A_{p / q}}+O\left(\varepsilon^{(\bar{m}+1) / 2}\right) .
$$

There are alternative ways to work out the resonant normal forms related to periodic orbits. We can compute the image of the $q$-th iteration of the map (2-3); in this case we must carry out the composition of trigonometric functions using the properties of the Bessel function. Given the explicit computation of $F^{q}$ we can use a fixed-point-like procedure to obtain the resonant normal form. See Appendix C.

\section{RULES TO DETERMINE THE RESONANT NORMAL FORM}

The amplitude of resonance of any hyperbolic periodic orbit with rotation number $p / q$ can be obtained if we compute its resonant normal form. In general, it is hard to solve a long sequence of homological equations to obtain the exponent $\bar{m}$ and the coefficient $A_{p / q}$. However, there is an easier procedure for finding $\bar{m}$, as follows:

1. From equation $(2-1)$, collect the set of exponents $\left\{a_{i}\right\}$ and the corresponding frequencies $\left\{w_{i}\right\}$, for $i=1, \ldots, N$. 
2. Let $\left\{n_{i}\right\}$ be any set of $N$ integers satisfying the condition

$$
q=\sum_{i=1}^{N} n_{i} w_{i} .
$$

3. Define the natural number

$$
m=\sum_{i=1}^{N}\left|n_{i}\right| a_{i} .
$$

4. Find the set of integers $\left\{n_{i}\right\}$ that minimize $m$. This minimum value of $m$ is the exponent $\bar{m}$. In this case we must suppose that $A_{p / q} \neq 0$.

To show how this algorithm works, we follow the construction of the resonant normal form step by step. From $(2-1)$, we have the set of exponents $\left\{a_{i}\right\}, i=1, \ldots, N$. This set can be ordered and so written in the form $\left\{\hat{a}_{i}\right\}, i=1, \ldots, N$, where $\hat{a}_{i}<\hat{a}_{i+1}$. Let the set of corresponding harmonic frequencies be $\left\{\hat{w}_{i}\right\}$. The left-hand side of the homological equation (2-6) can be expressed as a power series in $\varepsilon$, each term of which will be a product of terms $\left(\varepsilon^{j} g_{j, k} e^{ \pm 2 \pi i k \theta}\right)^{n}$. The lowest-order term of this series is $\varepsilon^{\hat{a}_{1}} g_{\hat{a}_{1}, \hat{w}_{1}} e^{ \pm 2 \pi i \hat{w}_{1} \theta}$. The next term could be

$$
\varepsilon^{\hat{a}_{2}} g_{\hat{a}_{2}, \hat{w}_{2}} e^{ \pm 2 \pi i \hat{w}_{2} \theta}
$$

or any product of the first term if $\hat{a}_{2} \geq n \hat{a}_{1}$, where $n$ is a positive integer. In this case the corresponding harmonic frequency is given by $\hat{w}_{2}$ or $\pm n \hat{w}_{1}$. The following term could be $\varepsilon^{\hat{a}_{3}} g_{\hat{a}_{3}, \hat{w}_{3}} e^{ \pm 2 \pi i \hat{w}_{3} \theta}$ or any product of previous term if $\hat{a}_{3} \geq n \hat{a}_{1}+m \hat{a}_{2}$, where $n$ and $m$ are positive integers. The related harmonic frequency is then given by $\hat{w}_{3}$ or $\pm n \hat{w}_{1} \pm m \hat{w}_{2}$. We can continue in a similar way to find the higher-order terms of this power series.

We can see from this procedure that any harmonic frequency $w$ will appear the first time when we can find a linear combination of the harmonic terms $\left\{w_{1}, w_{2}, \ldots, w_{N}\right\}$ such that

$$
w=\sum_{j=1}^{N} n_{j} w_{j} .
$$

The set of integers $n_{j}$ is chosen to minimize the sum $m_{w}=\sum_{j=1}^{N}\left|n_{j}\right| a_{j}$. Given the value of $m_{w}$ we know that the harmonic terms of frequency $w$ are of order $O\left(\varepsilon^{m_{w}}\right)$.

To compute the value of $A_{p / q}$ we must find the resonant normal form explicitly, for which one needs an algebraic manipulator. An alternative way to estimate the value of $A_{p / q}$ for any resonant normal form is to use a numerical procedure to compute the eigenvalues of the hyperbolic periodic points with rotation number $p / q$, when the value of the parameter goes to zero. Given the values of $\lambda_{p / q}$ and $\bar{m}$, we can find the value of $A_{p / q}$ using equations (2-10) and $(2-11)$. The numerical algorithm to find the hyperbolic periodic points can be reduced to a onedimensional method if our periodic orbits are monotone.

\section{NUMERICAL COMPUTATION}

In the last section we developed asymptotic methods to obtain the amplitude of any resonance. Now, we want to compare these results with the numerical computation of the amplitude of resonance. From a formal point of view, asymptotic methods can be applied only for a very small value of the perturbation parameter. Thus the numerical methods must be implemented with high precision arithmetic, because the linear behavior close to the periodic orbits has extremely slow dynamics since the eigenvalues of the periodic points are proportional to a power of the perturbation parameter; see $(2-11)$.

The main idea to compute the amplitude of resonance is to find the invariant manifolds of the hyperbolic periodic orbits related to our resonance. A naive method to compute the invariant manifolds is to find the eigenvectors of the periodic orbits; then we can iterate points which are located in the direction of the eigenvectors. But these points must be chosen very close to the periodic point. In this way we can expand the unstable invariant manifold by iterating these points a sufficient number of times; for the stable manifold we use the inverse map. This method is very inefficient for small values of the parameter: we must iterate a great number times in order to escape from the neighborhood of the periodic orbit.

An alternative method is to find a better approximation of the local invariant manifolds, representing them as the graph of a polynomial function. In that way we can choose points that belong to the graph but far from the periodic point. The computation of the local invariant manifolds gives a numerical method to obtain the resonance of any periodic point. We can use this method to find the maximum 
distance between the manifolds (measured orthogonally to the line that joins the periodic point with its next image). In that way we obtain a numerical computation of the amplitude of resonance.

We now describe how we can compute the invariant manifolds and the amplitude of resonance:

1. Finding Periodic Orbits. The first problem is to find a periodic orbit with specific rotation number $p / n$. This is a two-dimensional problem, so we must use a two-dimensional root finder to locate the periodic orbit. However, our map can be written as the product of two involutions. For two-dimensional maps with this property we can find periodic orbits by looking for specific curves, known as symmetry lines, that correspond to the invariant curves of the involutions. This is useful because it lets us reduce the problem of finding periodic orbits to a one-dimensional problem. A complete description of this procedure can be found in [Greene 1979].

2. Finding Invariant Manifolds. Once the periodic orbit has been found, we can compute its eigenvalues and eigenvectors, and so determine the local invariant manifolds. A first approximation of these invariant sets is the straight line segment starting at the periodic point and parallel to the corresponding eigenvector. This linear approximation is not enough for our purposes; we must find a high-order approximation. Write the map as

$$
\left(x_{n+1}, y_{n+1}\right)=H\left(x_{n}, y_{n}\right) .
$$

Let $\left(x_{p}, y_{p}\right)$ be our periodic point with rotation number $p / n$. This is a fixed point of the $q$-th iterate of our map:

$$
\left(x_{p}, y_{p}\right)=H^{n}\left(x_{p}, y_{p}\right)=H\left(H\left(\cdots\left(H\left(x_{p}, x_{p}\right)\right)\right)\right) .
$$

Now we must find the Taylor expansion of the $x$ and $y$-components of the map $H^{n}$ around the periodic point. Let $\tilde{H}(\hat{x}, \hat{y})$ be the Taylor series (understood componentwise) of the map (4-1), where the new variables are $\hat{x}=x-x_{p}$ and $\hat{y}=y-y_{p}$. We can compute the second iteration of (4-1) using our polynomial approximation:

$$
\tilde{H}^{2}(\hat{x}, \hat{y})=\tilde{H}(\tilde{H}(\hat{x}, \hat{y})) .
$$

Then we can compute the Taylor expansion of this second iterate. We know that the components of $\tilde{H}$ are polynomials of degree $M$; the composition
$\tilde{H}(\tilde{H}(\hat{x}, \hat{y}))$ should be computed as a polynomial of degree $M$ as well. This can be done using symbolic routines to add, multiply and truncate polynomial expansions.

In this way we obtain an expression for the map around the periodic point which can be written as a polynomial of degree $M$. We can repeat this procedure $n$ times to obtain a polynomial approximation of the map (4-2). In our new set of coordinates, the origin is a fixed point of the map:

$$
\left(\hat{x}_{n+1}, \hat{y}_{n+1}\right)=\tilde{H}^{n}\left(\hat{x}_{n}, \hat{y}_{n}\right) .
$$

Now let $\tilde{F}^{n}$ and $\tilde{G}^{n}$ be the $\hat{x}$ - and $\hat{y}$-components of the iterate $\tilde{H}^{n}$. The next step is to describe the invariant manifolds as the graph of a function of one variable $\varphi(\hat{x})=\hat{y}$. This function can be approximated by a polynomial of degree $M$. Let $\varphi(\hat{x})$ be the graph describing the local invariant manifold. We know that the image of any point of the invariant manifold must belong to the invariant manifold. Therefore

$$
\varphi\left(\tilde{F}^{n}(\hat{x}, \varphi(\hat{x}))\right)=\tilde{G}^{n}(\hat{x}, \varphi(\hat{x})) .
$$

Because $\tilde{F}^{n}$ and $\tilde{G}^{n}$ are polynomials of degree $M$, we can manipulate this equation in order to determine the value of the unknown coefficient that defines the polynomial function $\varphi(\hat{x})$ up to degree $M$.

We now define the fundamental interval. This set belongs to the unstable invariant manifold and equals $\left\{(\hat{x}, \varphi(\hat{x})): \hat{x} \in\left[\hat{x}_{0}, \tilde{F}^{n}\left(\hat{x}_{0}, \varphi\left(\hat{x}_{0}\right)\right)\right]\right\}$, where $\left|\hat{x}_{0}\right| \ll 1$. The stable invariant manifold has a similar expression. The successive images of the fundamental interval form the local invariant manifold. We want to extend the invariant manifold until this curve arrives close to the next image of our periodic orbit. In order to select a suitable value of $\hat{x}_{0}$, we must take in to account that the residue of $\varphi(\hat{x})$ is of order $O\left(\hat{x}_{0}^{M+1}\right)$. This approximation allows us to choose values of $\hat{x}_{0}$ greater than the linear approximation of the invariant manifold in some order of magnitude.

3. Amplitude of Resonance. To find the amplitude of resonance, we set the periodic point $\boldsymbol{x}_{0}$ and we also find the nearest image of this point in phase space, this point is denoted by $\boldsymbol{x}_{1}$. Now, we have computed the approximation of the stable and unstable invariant manifolds of our periodic point, $\mathcal{W}^{s}\left(\boldsymbol{x}_{0}\right)$ and $\mathcal{W}^{u}\left(\boldsymbol{x}_{0}\right)$. Let $\overline{S P}$ be the line segment that joins 
the points $\boldsymbol{x}_{0}$ and $\boldsymbol{x}_{1}$, parametrized by the function $s p(t)$, where $t \in[0,1]$. We define $\overline{S W}(t)$ as the line segment that is orthogonal to $\overline{S P}$ at the point $s p(t)$ and has initial point located in $\mathcal{W}^{s}\left(\boldsymbol{x}_{0}\right)$ and its final point in $\mathcal{W}^{u}\left(\boldsymbol{x}_{0}\right)$. The amplitude of resonance is defined as the maximum length of $\overline{S W}(t)$ for $t \in[0,1]$.

A more complete description of the construction of invariant manifolds using numerical and asymptotic methods can be found in [Simó 1990].

An important question for any resonance is to find how the amplitude of resonance depends to the perturbation parameter $\varepsilon$. To obtain this relation, we can compute the amplitude of resonance for a sequence of values of the parameter $\left\{\varepsilon_{i}\right\}$ such that $\varepsilon_{i+1}<\varepsilon_{i}$ for $i=0, \ldots, L$. Given any value of $\varepsilon_{i}$, we can compute the corresponding amplitude of resonance $\Delta_{i}$. We then compute a linear regression of the set of values $\left(\log \varepsilon_{i}, \log \Delta_{i}\right)$, for $i=0, \ldots, L$. This gives us a functional relation between the parameter and the amplitude of resonance, of the form

$$
\Delta=\beta \varepsilon^{\alpha} .
$$

Our numerical procedure allows us to compute the invariant manifolds with a very high precision, which depends on the order of the polynomial function $\varphi(\hat{x})$. To compute this function with an error less than $10^{-22}$, we calculated the polynomial function up to degree 30 and used quadruple-precision arithmetic.

At this point, we can compare the amplitude of any resonance as computed by the resonant normal form of Section 2 and by the numerical procedure just described, and so get an idea the accuracy of the asymptotic procedure. We did this for three rotation numbers; in these examples the perturbation function has only one harmonic, $\varepsilon f(x, \varepsilon)=$ $\varepsilon /(2 \pi) \sin (2 \pi x)$ in equation $(2-1)$. Here are the values obtained; we can see that agreement is good:

\begin{tabular}{|ccc|}
\hline$p / n$ & $\Delta_{p / n}$ normal form & $\Delta_{p / n}$ numerical \\
\hline $1 / 3$ & $\frac{\sqrt{2}}{12 \pi} \varepsilon^{3 / 2} \approx 0.037513 \varepsilon^{3 / 2}$ & $0.037538 \varepsilon^{1.5009}$ \\
$1 / 4$ & $\frac{\sqrt{15}}{48 \pi} \varepsilon^{2} \approx 0.025685 \varepsilon^{2}$ & $0.025684 \varepsilon^{1.9999}$ \\
$1 / 6$ & $\frac{1}{2 \pi} \sqrt{\frac{33}{1280}} \varepsilon^{3} \approx 0.02555 \varepsilon^{3}$ & $0.02549 \varepsilon^{2.996}$ \\
\hline
\end{tabular}

The resonant normal form $(2-10)$ is not easy to obtain for periodic orbits with large period. This is because the number of terms that we must work out from this asymptotic method increases like a combinatorial problem. However, we showed at the end of Section 3 a procedure to estimate the amplitude of resonance without computing the complete resonant normal form. This is an asymptotic method and it is valid for asymptotically small values of the perturbation parameter. For any perturbation function $(2-1)$ and rotation number $p / q$, we can compute the corresponding amplitude of resonance if we are able to find the eigenvalues of the hyperbolic periodic points using equations $(2-10)$ and $(2-11)$. To obtain the value of $\bar{m}$, we can follow the rules described in Section 4. It is easy to find the numerical value of $\lambda_{p / q}$ for small values of $\varepsilon$. The first step of our numerical procedure describes how we can find hyperbolic periodic points and their eigenvalues. Hence, we can estimate the coefficient $A_{p / q}$ of the resonant normal form:

$$
A_{p / q}=\varepsilon^{-\bar{m}} \frac{\left(\lambda_{p / q}-1\right)^{2}}{2 \pi q} .
$$

We must be careful about how small we must choose the value of the perturbation parameter $|\varepsilon|$ for periodic orbits with large period. This is because the numerical procedure which computes the eigenvalue of any orbit can reach an arithmetic underflow in our machine and then we can loose the precision of the numerical computation.

In the next sections we show many examples where we had to compute the amplitude of resonance; in all these cases we used the method to compute the eigenvalues in order to estimate the coefficients $\Delta$ in equation (4-3).

\section{ESTIMATE OF $\Delta_{\mathrm{p} / \mathrm{q}}$ FOR VALUES OF $\varepsilon$ OF THE ORDER OF 1}

We now show how we can use the asymptotic behavior of the amplitude of resonances in order to find a good estimate of $\Delta_{p / q}$ when $\varepsilon$ is of the order of 1 . The idea is that only few harmonics of the perturbation function (2-1) provide the main contribution to the size of the amplitude of resonance. We found that the asymptotic behavior of different sets of harmonics determine the amplitude for specific intervals of values of $\varepsilon$ that can be far from 0 . 
We present two simple examples where we show the form that we can determine, for any range of values of $\varepsilon$, which is the dominant harmonic of $(2-1)$. From these examples we express a more general conjecture about the behavior of the amplitude of resonance.

Example 1. Let the perturbation function be

$$
f(x)=\frac{\varepsilon}{2 \pi}\left(\sin (2 \pi x)+10^{-\alpha} \sin (2 \pi(9 x)),\right.
$$

where $\alpha$ will be taken equal to 9,15 and 21. Suppose we want to find the growth rate of $\Delta_{2 / 9}$ for a periodic orbit with rotation number $\frac{2}{9}$. Using the rules given in Section 3 , we get $\bar{m}=1$. Then the asymptotic behavior can be determined with the help of equation $(2-10)$. This relation is similar for the three values of the exponent $\alpha$ (though $A_{2 / 9}$ depends on the value of $\alpha$ ). However, the numerical computation of $\Delta_{2 / 9}$ shows that the asymptotic behavior is not accurate for $\varepsilon$ greater than $10^{-3}$.

The results are summarized in Figure 3, which plots $\log _{10} \varepsilon$ horizontally and $\log _{10} \Delta_{2 / 9}$ vertically. The three curves F1, F2, F3 correspond to $\alpha$ equal to 9,15 and 21 respectively. For small values of $\varepsilon$, say $|\varepsilon|<10^{-3}$, these curves look like straight lines of slope $\frac{1}{2}$. Hence the asymptotic estimate of $\Delta_{2 / 9}$ agrees with the numerical computation (lines B, C and $\mathrm{D})$. For higher values of $\varepsilon$, each curve changes its slope to $\frac{9}{2}$, so the amplitude then grows as $\Delta_{9 / 2} \sim$ $\varepsilon^{9 / 2}$. For $\varepsilon$ greater than 0.1 , the three curves behave like straight lines with slope $\frac{9}{2}$.

We can compare these results with a similar example in which we take into account only the first harmonic of the perturbation function:

$$
f(x)=\frac{\varepsilon}{2 \pi}(\sin 2 \pi x) .
$$

The asymptotic behavior of this case corresponds to a straight line with slope $\frac{9}{2}$ (line A in Figure 3). Next, we can do a similar asymptotic computation but now with a perturbation function that has only the second harmonic of $(5-1)$ :

$$
f(x)=\frac{\varepsilon}{2 \pi} 10^{-\alpha} \sin (2 \pi(9 x)) .
$$

We obtain three lines B, C, D with slope $\frac{1}{2}$, corresponding to $\alpha=9,15$ and 21. The intersections of these lines represent the transition points where the curve of the amplitude of resonance changes slope.

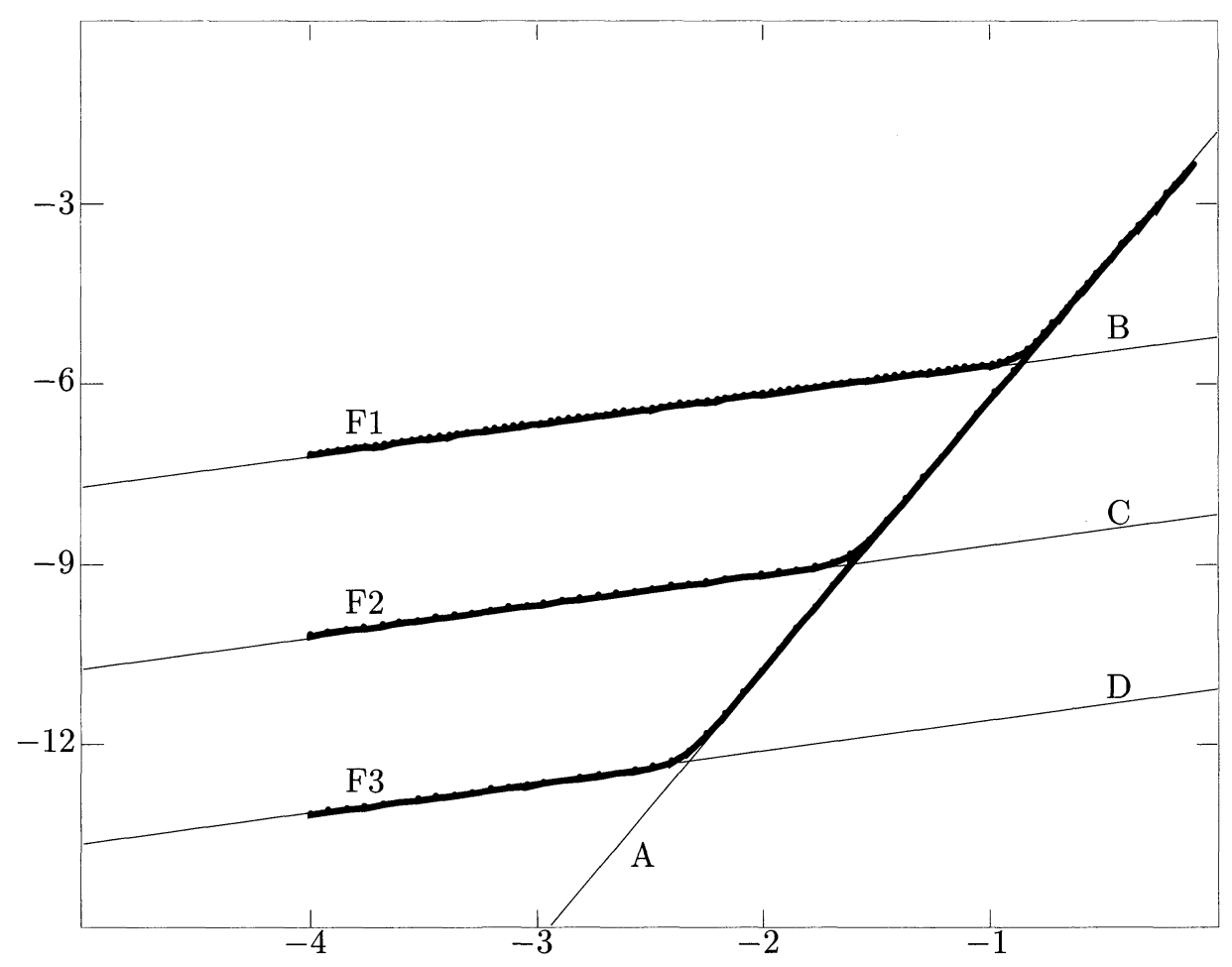

FIGURE 3. Graphs of $\log _{10} \Delta_{2 / 9}$ as a function of $\log _{10} \varepsilon$. The bold curves F1, F2 and F3 correspond to $\Delta_{2 / 9}$ with $\alpha$ equal to 9,15 and 21 . The lines $\mathrm{B}, \mathrm{C}$ and $\mathrm{D}$ are the asymptotic behavior of (5-1) with $\alpha$ equal to 9,15 and 21. Line $\mathrm{A}$ is obtained using the first harmonic in the perturbation function. 
We can also see in Figure 3 that these straight lines represent a lower bound for the rate of growth of the amplitude of resonance. In fact, these straight lines form a convex hull of the amplitude of resonance.

Example 2. This is similar to the first example, but we include an additional harmonic on the perturbation function:

$$
\begin{aligned}
f(x)=\frac{\varepsilon}{2 \pi}(\sin (2 \pi x)+ & 10^{-4} \sin (2 \pi(3 x)) \\
+ & \left.10^{-21} \sin (2 \pi(9 x))\right) .
\end{aligned}
$$

Now we want to find the growth rate of the amplitude of resonance for a periodic orbit with rotation number $\frac{4}{9}$. The numerical computation of $\Delta_{4 / 9}$ is shown in Figure 4. In this example we can see three different behaviors of the rates of growth of the amplitude: For small values of $\varepsilon$, the curve looks like a straight line; this part of the curve is dominated by the third harmonic of (5-3). For $\varepsilon$ in the interval $10^{-4}<\varepsilon<10^{-2}$, the second harmonic of $(5-3)$ dominates. Finally, for $\varepsilon>10^{-2}$, the dominant harmonic is the first.

As in the first example, we can find the convex hull (lower bound) for the curve of $\Delta_{4 / 9}$. This lower bound is given by the amplitude of the resonances obtained from the perturbation function when we consider only one harmonic. In this case we can compute the asymptotic behavior of $\Delta_{4 / 9}$ taking only one harmonic from the perturbation function $(5-3)$ :

$$
\begin{aligned}
& f_{A}(x)=\frac{\varepsilon}{2 \pi} \sin (2 \pi x) \\
& f_{B}(x)=\frac{\varepsilon}{2 \pi} 10^{-4} \sin (2 \pi(3 x)), \\
& f_{C}(x)=\frac{\varepsilon}{2 \pi} 10^{-21} \sin (2 \pi(9 x)) .
\end{aligned}
$$

Figure 4 shows three straight lines which correspond to the rate of growth of the three harmonics, that is, when we take only one harmonic in the perturbation function. We observe again that the curve which represents $\Delta_{4 / 9}$ for the perturbation function $(5-3)$, is located over the three straight lines and the values of $\varepsilon$, where the curve changes its slope, can be estimated by the intersection points of the three straight lines.

From these two examples, we can formulate a more general conjecture:

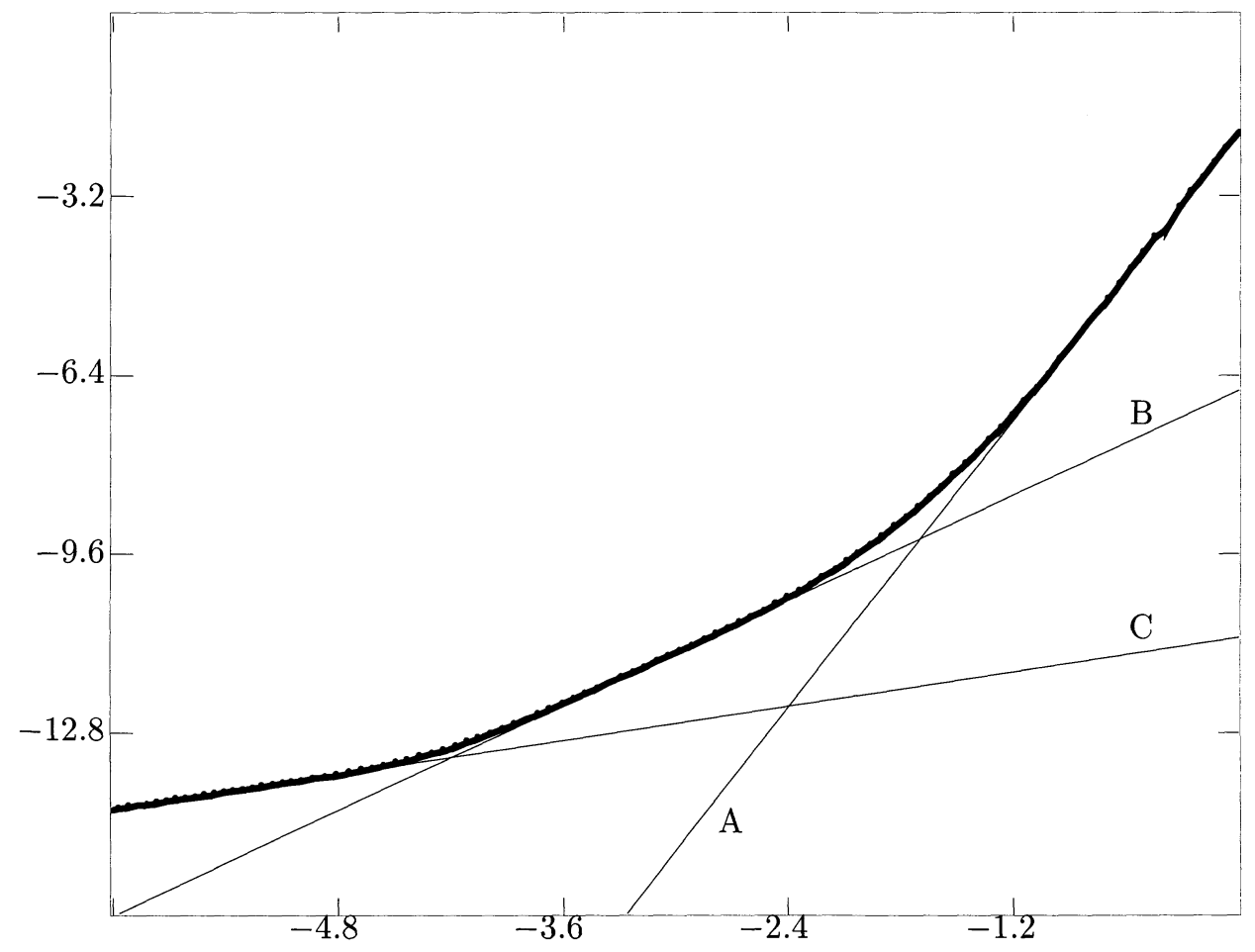

FIGURE 4. Axes are described in Figure 3. The bold curve correspond shows $\Delta_{4 / 9}$ computed via (5-3). Lines A, $\mathrm{B}$ and $\mathrm{C}$ show $\Delta_{4 / 9}$ computed using only the first, second and third harmonic respectively in (5-3). 
Lower Bound Conjecture. Let $\varepsilon f(x, \varepsilon)$ be an analytic perturbation function. For any rational rotation number $p / q$, we can estimate a lower bound for the amplitude of the resonance with this rotation number as follows:

1. Using the Fourier series of the perturbation function, we truncate this series at $N \leq q$. We consider only those harmonics such that the integer $q$ can be written as an integer combination of them.

2. For any set of selected harmonics, we compute their asymptotic behavior. In this step we can follow the rules described in Section 3.

3. From each set of harmonics we plot the corresponding line of $\log \Delta_{p / q}$ versus $\log \varepsilon$. This set of lines forms a convex hull representing a lower bound for the amplitude of resonance of the actual perturbed twist map.

4. The transition points, where there is a change in the slope of the amplitude of resonance curve, can be estimated by the intersection of the straight lines.

\section{COLLAPSE OF RESONANCE}

The numerical computation of amplitudes of resonance for different kinds of perturbation functions allows us to find the relation between the value of the perturbation parameter and the amplitude of resonance for any rotation number. Usually, the amplitude of resonance behaves as a monotone function of the perturbation parameter, but there are perturbation functions for which this is not so; in such cases, the amplitude decays to zero in a certain interval of values of $\varepsilon$ and later grows again to resume its previous behavior (see Figure 5 for an example). We now consider this phenomenon, which we call the collapse of resonance, and give for it a simple explanation in terms of the different harmonics that make up the perturbation function.

Collapse of resonance happens when the contributions of two or more harmonics of the perturbation function are out of phase in some small domain of the parameter. In this case the total contribution to the amplitude is null and the size of this resonance goes to zero. We have this situation when two harmonics of the perturbation function have opposite sign and they behave as the principal contribution to the amplitude. We can estimate the range of values

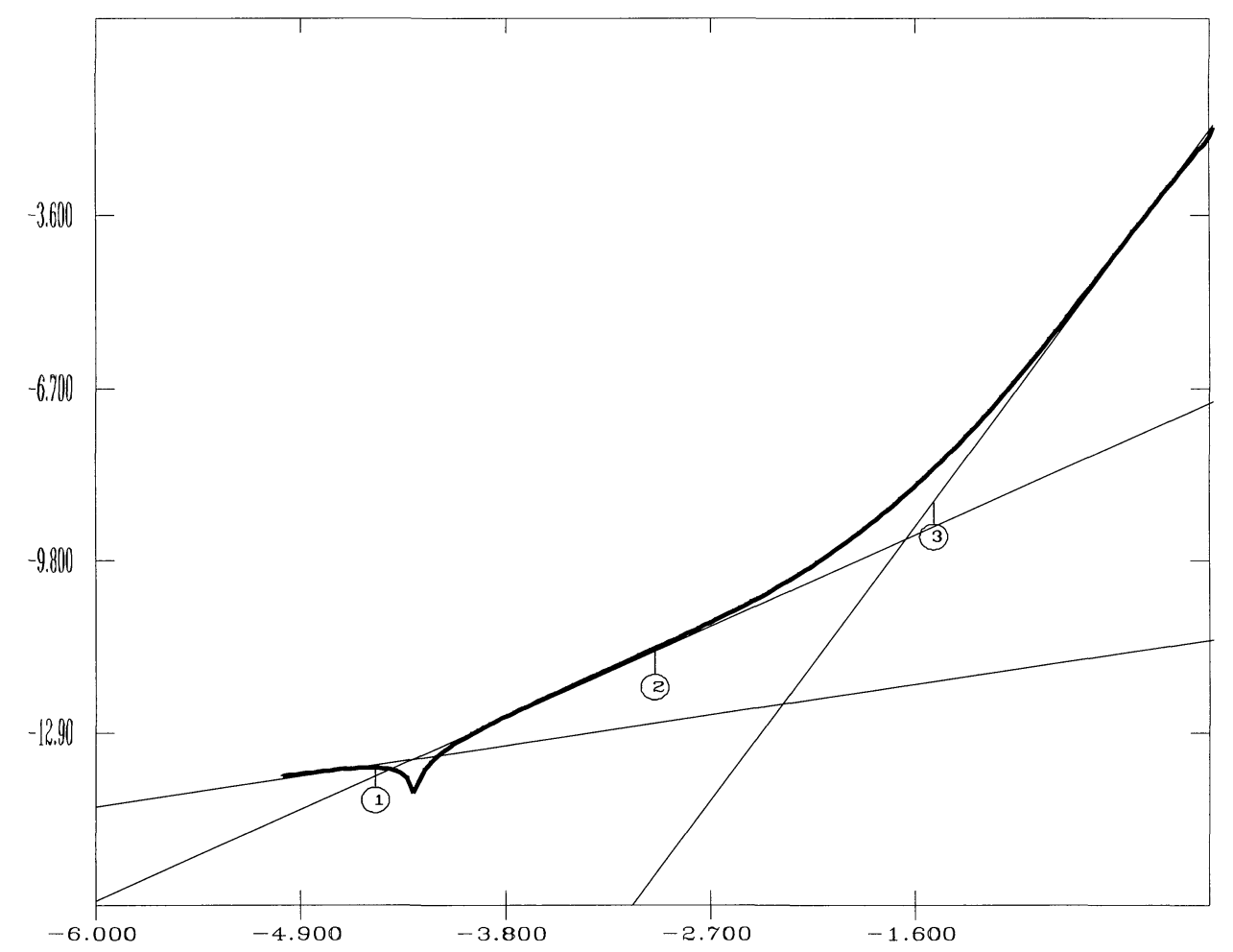

FIGURE 5. Rotation number: $\frac{4}{9}$. Lines 1,2 , and 3 correspond to harmonics 9,3 , and 1 , respectively. 
of $\varepsilon$ where we expect to find collapse; this situation takes place when the contribution of the different harmonics of the perturbation function are of the same order of magnitude. The asymptotic behavior of these harmonics corresponds to straight lines in a log-log graph. For any two harmonics which have opposite sign, the intersection point of the two lines, represents the value of the parameter where the resonance could collapse.

The next examples show how we can predict the collapse of a specific resonance. Consider the perturbation function

$$
\begin{aligned}
\varepsilon f_{1}(x)=\frac{\varepsilon}{2 \pi} & (\sin (2 \pi x) \\
& \left.+10^{-4} \sin (2 \pi(3 x))-10^{-21} \sin (2 \pi(9 x))\right) .
\end{aligned}
$$

Figure 5 shows the numerical computation of the amplitude of resonance $\Delta_{4 / 9}$ and the lines corresponding to the asymptotic behavior of each separate harmonic. We see that the amplitude collapses in the region of parameters where line 1 (corresponding to the ninth harmonic) and line 2 (third harmonic) intersect. In this case we have collapse because the ninth and third harmonics have opposite sign and similar magnitude.
Next we take the perturbation function

$$
\begin{aligned}
\varepsilon f_{2}(x)= & \frac{\varepsilon}{2 \pi}(\sin (2 \pi x) \\
& \left.\quad-10^{-4} \sin (2 \pi(3 x))-10^{-21} \sin (2 \pi(9 x))\right) .
\end{aligned}
$$

The graph of the numerical computation of $\Delta_{4 / 9}$ is shown in Figure 6, together with the lines corresponding to the asymptotic behavior of the three harmonics. In this case the collapse occurs in the neighborhood of the intersection of lines 2 and 3 (the latter corresponds to the first harmonic). We can predict this collapse because the first and third harmonics have opposite sign.

The Lower Bound Conjecture can be extended to encompass the collapse of resonance:

Collapse of Resonance Conjecture. The intersection of two lines corresponding to harmonics with opposite sign gives the value of the parameter where we expect collapse of resonance.

An important fact related to the collapse of resonance phenomenon is that the left branch of the amplitude of resonance, with respect to the point where the amplitude has collapsed, corresponds to a periodic orbit different from the periodic orbit produced

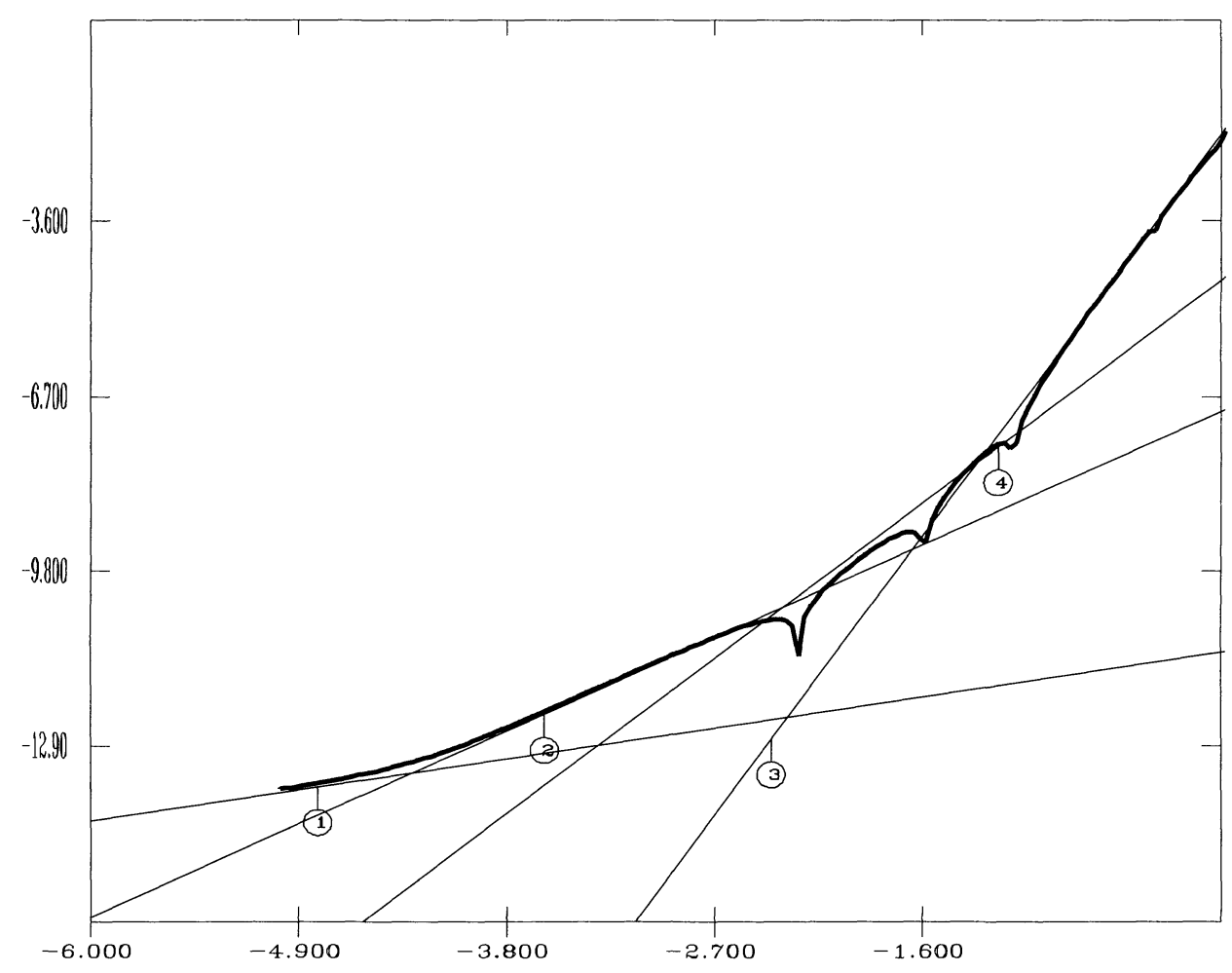

FIGURE 6. Rotation number: $\frac{4}{9}$. Lines 1,2 , and 3 correspond to harmonics 9,3 , and 1 , respectively. Line 4 corresponds to harmonics 1 and 3 . 
by the right branch of the curve. Dynamically, the collapse of resonance is related to one periodic orbit whose amplitude of resonance is decreasing in some interval of the parameter; the amplitude decreases until the resonance disappears: this is because the hyperbolic periodic orbit collides with the elliptic orbit that shares the same rotation number. This phenomenon corresponds to a saddle-node bifurcation, which in turn corresponds to a creation or destruction of a set of periodic orbits. Simultaneously, a pair of periodic orbits with the same rotation number, is created by the mechanism of the saddle-node bifurcation: the amplitude of resonance of the corresponding hyperbolic periodic orbit grows from zero and behaves like a step function. The Birkhoff twist theorem shows that any twist map has at least one pair of periodic orbits for any rational rotation number belonging to the rotation interval of the map. Usually, we only have one pair of periodic orbits for any value of the perturbation parameter. Collapse of resonance is a case where we can find two pairs of periodic orbits sharing the same rotation number and existing simultaneously for a small open set of values of the perturbation parameter.

\section{AN EXAMPLE OF ANALYTICAL PERTURBATION}

We now show an example where we compute the amplitude of resonance for an analytic perturbation function. Consider the perturbation function

$$
\varepsilon f(x)=\varepsilon \sin (a \cos (2 \pi x)) .
$$

We can see that $f(x)$ is an analytic periodic function and the average of this function is 0 . Suppose that we want to study the resonance $3 / 10$ of this problem in a range of values of the perturbation parameter $\varepsilon \in[0,1]$. We fix the parameter $a=0.01$.

The first step is to find the Fourier expansion of the perturbation function. This is easy to do if we use some properties of Bessel functions [Abramowitz and Stegun 1972]:

$$
\sin (a \cos x)=2 \sum_{k=0}^{\infty} J_{2 k+1}(a) \sin (2 \pi(2 k+1) x), \quad(7-1)
$$

where $J_{i}(a)$ are the Bessel functions of integer order $i$. Because we are interested in the resonance 3/10, we must cut the Fourier series at harmonic 10:

$$
\varepsilon f(x)=2 \varepsilon \sum_{k=0}^{4} J_{2 k+1}(a) \sin (2 \pi(2 k+1) x) .
$$

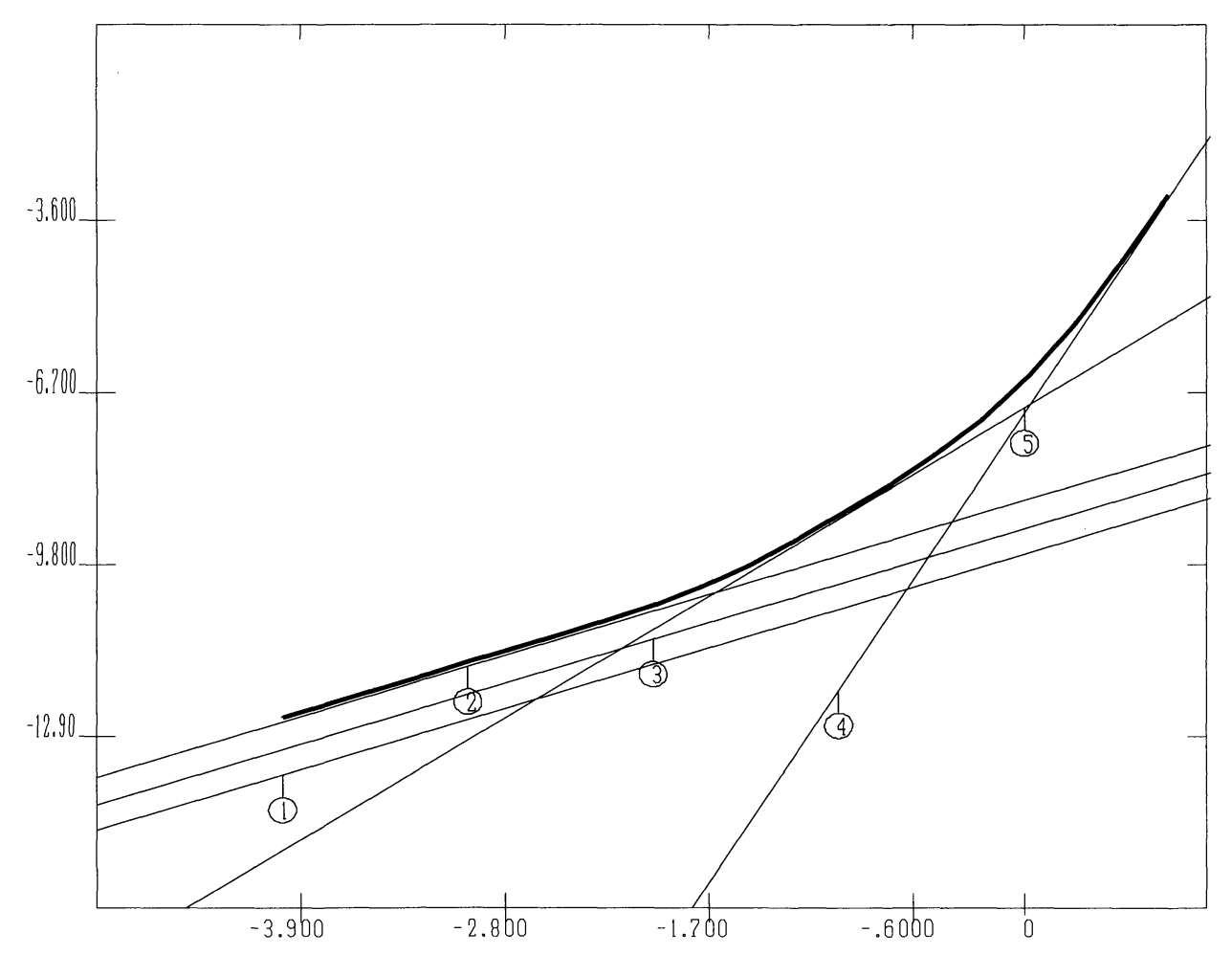

FIGURE 7. Rotation number: $\frac{3}{10}$. Line 1 : harmonics 1 and 9 ; line 2: harmonics 3 and 7; line 3 : harmonic 5 ; line 4: harmonic 1 ; line 5 : harmonics 1,3 , and 5 . 
We only have harmonics $1,3,5,7$, and 9 , and we can find five linear combinations of these harmonics in order to reach harmonic 10 . The next step is to determine the asymptotic behavior of each linear combination. The results are:

\begin{tabular}{|crc|}
\hline Harmonics & $\bar{m}$ & $A_{3 / 10}^{\text {asympt }}$ \\
\hline 1 and 9 & 1 & $3.6164 \times 10^{-10}$ \\
3 and 7 & 1 & $3.2794 \times 10^{-9}$ \\
5 & 1 & $1.0384 \times 10^{-9}$ \\
1 & 10 & $1.2244 \times 10^{-7}$ \\
1,3 and 5 & 4 & $1.5325 \times 10^{-7}$ \\
\hline
\end{tabular}

We can verify that the coefficients of the perturbation function (7-2) are positive, that is $J_{i}(a)>0$ for $a=0.01$. This means that we do not expect to have any collapse of resonance, in this case the amplitude of resonance must behave as a monotone function. Figure 7 shows the numerical computation of amplitude of resonance $\Delta_{3 / 10}$ with perturbation function (7-1). The five straight lines correspond to the asymptotic behavior of the harmonics given above. This set of straight lines determines a good estimate of $\Delta_{3 / 10}$. The range of values of the perturbation parameter in our example is $\varepsilon \in(0,3.163)$.

Note that we have obtained a good estimate of $\Delta_{3 / 10}$ using only asymptotic information on the linear combination of five harmonics, and these asymptotic behaviors were computed for values of the parameter close to zero.

\section{CONCLUSION}

We have presented a simple algorithm for studying the nonlinear behavior of any resonance of twist maps. We gave several examples of how to predict the amplitude of resonance given a rational rotation number. In each case, we only needed to know the asymptotic behavior of the standard map and we used a simple perturbation function composed of a few terms of the Fourier expansion of the initial perturbation function. The hardest task was determining the eigenvalue of the hyperbolic periodic orbit having the same rotation number when the value of the parameter is asymptotically small. Using this information, we could obtain a good set of lower bounds for the amplitude of resonances and we could predict the collapse of resonances.
Using only linear information for small values of the parameter, we were able to predict the nonlinear behavior of the map when the perturbation parameter is of order 1.

The perturbation functions we have used in our previous examples are written as sums of sine functions. The main reason to use only odd functions is related to our numerical method to compute periodic orbits. When the General Standard Map is written with an odd perturbation function, this map can be described as the composition of two maps, each of which is an involution, and it has a pair of invariant curves in the plane. One can show that we can find periodic orbits which have points in these invariant curves, so we only need to look for the periodic orbits, for any rotation number, on the symmetry lines. Using this method, we can simplify significantly the numerical procedure to find periodic orbits because we are able to find these orbits using a one-dimensional root-finder. Nevertheless, the method to estimate the amplitude of resonance can be used for any perturbation function.

\section{APPENDIX A: HAMILTONIAN FLOWS AND SYMPLECTIC TWIST MAPS}

Small perturbations of the integrable Hamiltonian system gives rise to more complex dynamics in phase space. A suitable way to perturb an integrable twist map is using a generating function; in this way we can assure that the transformed map has the same symplectic structure. Any integrable twist map can be written in the form

$$
r_{i+1}=r_{i}, \quad \varphi_{i+1}=\varphi_{i}+\alpha\left(r_{i+1}\right),
$$

where $\left(\varphi_{i}, r_{i}\right) \in S^{1} \times \mathbb{R}$ and $i \in \mathbb{Z}$. In this case the value of the function $\alpha(r)$ coincides with the rotation number of the orbit and is a monotone function; from now on we set $d \alpha(r) / d r>0$. The generating function of the map just defined is

$$
G\left(r_{i+1}, \varphi_{i}\right)=r_{i+1} \varphi_{i}+B\left(r_{i+1}\right),
$$

where $d B(r) / d r=\alpha(r)$. Let $g(r, \varphi, \varepsilon)$ be an analytic function which is periodic with respect to $\varphi$. Then we can perturb the expression of the generating function as follows:

$$
G\left(r_{i+1}, \varphi_{i}\right)=r_{i+1} \varphi_{i}+B\left(r_{i+1}\right)+\varepsilon g\left(r_{i+1}, \varphi_{i}, \varepsilon\right) .
$$


Define the functions

$$
f_{1}(r, \varphi, \varepsilon)=\frac{\partial g}{\partial \varphi}, \quad f_{2}(r, \varphi, \varepsilon)=\frac{\partial g}{\partial r} .
$$

Then our perturbed twist map is given by

$$
\begin{aligned}
r_{i+1} & =r_{i}+\varepsilon f_{1}\left(r_{i+1}, \varphi_{i}, \varepsilon\right), \\
\varphi_{i+1} & =\varphi_{i}+\alpha\left(r_{i+1}\right)+\varepsilon f_{2}\left(r_{i+1}, \varphi_{i}, \varepsilon\right) .
\end{aligned}
$$

This is in fact a one-parameter family of twist maps; the parameter is $\varepsilon$, and we assume that $|\varepsilon| \ll 1$. The functions $f_{1}$ and $f_{2}$ are periodic with respect the angular coordinate $\varphi$ and we impose the condition $\lim _{\varepsilon \rightarrow 0} \varepsilon f_{i}(r, \varphi, \varepsilon)=0$.

In order to study the dynamics around monotone periodic orbits of the perturbed map $(\mathrm{A}-1)$ it is convenient to transform it. The idea is to find a simple map which coincides with $(\mathrm{A}-1)$ in the neighborhood of specific rotation number. This transformation must be symplectic in order to preserve the Hamiltonian properties of the map $(\mathrm{A}-1)$. A way of carrying out symplectic transformation is to find a suitable generating function which can be used to construct the transformation. We want to transform the set of coordinates $(r, \varphi)$ into new coordinates $(\rho, \varphi)$ with the property $\rho_{i+1}=\alpha\left(r_{i+1}\right)+$ $\varepsilon f_{2}\left(r_{i+1}, \varphi_{i}, \varepsilon\right)$.

Using the implicit function theorem we can write $r_{i+1}$ as a function of $\varphi_{i}$ and $\rho_{i+1}$, then we obtain $r_{i+1}=\gamma_{1}\left(\rho_{i+1}, \varphi_{i}, \varepsilon\right)$. Because $\varepsilon$ is small, this function is then written as

$$
r_{i+1}=\alpha^{-1}\left(\rho_{i+1}\right)+\varepsilon \gamma_{2}\left(\rho_{i+1}, \varphi_{i}, \varepsilon\right) .
$$

Therefore our generating function is given by

$$
G\left(\rho_{i+1}, \varphi_{i}\right)=\alpha^{-1}\left(\rho_{i+1}\right) \varphi_{i}+\varepsilon \int_{\varphi_{i}} \gamma_{2}\left(\rho_{i+1}, s, \varepsilon\right) d s .
$$

Because the second equation of $(\mathrm{A}-1)$ is transformed to $\varphi_{i+1}=\varphi_{i}+\rho_{i+1}$, the variable $\varphi_{i}$ can be substituted in $(\mathrm{A}-2)$ :

$$
\begin{aligned}
r_{i+1} & =\alpha^{-1}\left(\rho_{i+1}\right)+\varepsilon \gamma_{2}\left(\rho_{i+1}, \varphi_{i+1}-\rho_{i+1}, \varepsilon\right) \\
& =\alpha^{-1}\left(\rho_{i+1}\right)+\varepsilon \hat{\gamma}_{2}\left(\rho_{i+1}, \varphi_{i+1}, \varepsilon\right) .
\end{aligned}
$$

This involves only coordinates with subindex $i+1$, so we can replace $i+1$ by $i$. Using (A-3), we can rewrite the first equation in $(\mathrm{A}-1)$ as follows:

$\alpha^{-1} \rho_{i+1}+\varepsilon \hat{\gamma}_{2}\left(\rho_{i+1}, \varphi_{i+1}, \varepsilon\right)$

$=\alpha^{-1} \rho_{i}+\varepsilon \hat{\gamma}_{2}\left(\rho_{i}, \varphi_{i}, \varepsilon\right)+\varepsilon f_{2}\left(\rho_{i}+\varepsilon \hat{\gamma}_{2}\left(\rho_{i}, \varphi_{i}, \varepsilon\right), \varphi_{i}, \varepsilon\right)$.
Replacing $\varphi_{i+1}$ by $\varphi_{i}+\rho_{i+1}$ and using again the implicit function theorem, we can write $\rho_{i+1}$ as a function of $\rho_{i}$ and $\varphi_{i}$. Therefore the new map is then given by

$$
\rho_{i+1}=\rho_{i}+\varepsilon \hat{\gamma}_{3}\left(\rho_{i}, \varphi_{i}, \varepsilon\right), \quad \varphi_{i+1}=\varphi_{i}+\rho_{i+1} .
$$

This must be a symplectic map, which means that its Jacobian has determinant 1 . This implies that the function $\hat{\gamma}_{3}\left(\rho_{i}, \varphi_{i}, \varepsilon\right)$ does not depend on $\rho_{i}$. The final form of map $(\mathrm{A}-4)$ is

$$
\rho_{i+1}=\rho+\varepsilon V\left(\varphi_{i}, \varepsilon\right), \quad \varphi_{i+1}=\varphi_{i}+\rho_{i+1} .
$$

We call this the General Standard Map. We must remark that the angular coordinate $\varphi$ was not transformed from (A-1) to (A-5), so the function $V(\varphi, \varepsilon)$ remains periodic with respect to the first argument, $V(\varphi+1, \varepsilon)=V(\varphi, \varepsilon)$.

The map (A-5) might not be a global representation of the dynamics of the map $(\mathrm{A}-1)$ because the domain of transformation $(\mathrm{A}-4)$ could be an open set of $\mathbb{R}$. The map (A-5) can then be used around a strip of the cylinder $S^{1} \times \mathbb{R}$. Therefore in order to study monotone periodic orbits we can choose the Standard Map as a general representation of a twist symplectic map.

\section{APPENDIX B: AMPLITUDE OF RESONANCE IN THE GENERIC CASE}

Consider the standard map (1-1); for small values of the parameter $\varepsilon$ we can estimate the eigenvalues of any monotone periodic orbit with rotation number $p / q$. Suppose that $V(x)$ is a $\mathrm{e}^{k}$ periodic function with $k \geq 2$. The map $F^{q}$ can be written in the neighborhood of periodic orbit, to first-order approximation, as follows [Veerman 1993]:

$$
\begin{aligned}
& y_{q}=y_{1}+\varepsilon \sum_{i=1}^{q-1} V\left(x_{i}+i y_{i}\right)+O\left(\varepsilon^{2}\right), \\
& x_{q}=x_{1}+q y_{1}+\varepsilon \sum_{i=1}^{q-1}(q-i) V\left(x_{i}+i y_{i}\right)+O\left(\varepsilon^{2}\right) .
\end{aligned}
$$

Using the coordinate transformation $y=p / q+$ $\varepsilon^{1 / 2} \zeta$, this becomes

$$
\begin{aligned}
& \zeta_{q}=\zeta_{1}+\varepsilon^{1 / 2} \sum_{i=1}^{q-1} V\left(x_{i}+i p / q\right)+O(\varepsilon), \\
& x_{q}=x_{1}+\varepsilon^{1 / 2} q \zeta_{1}+p+O(\varepsilon) .
\end{aligned}
$$


The eigenvalues of the fixed points $x_{1}=x_{q}-p$ and $\zeta_{q}=\zeta_{1}$ are

$$
\lambda_{ \pm}=1 \pm \varepsilon^{1 / 2}\left(\sum_{i=1}^{q-1} D V\left(x_{i}+i p / q\right)\right)^{1 / 2}+O(\varepsilon),
$$

where $D V$ is the derivate of $V$ and the set of values $\left\{x_{i}\right\}$ must be the solution of the equation

$$
\sum_{i=1}^{q-1} V\left(x_{i}+i p / q\right)=0 .
$$

Genericlly, the asymptotic value of the eigenvalues satisfies

$$
\left|\lambda_{ \pm}-1\right| \sim O\left(\varepsilon^{1 / 2}\right)
$$

therefore, the amplitude of resonance is given by the following relation, obtained from the pendulum equation:

$$
\Delta_{p / q}=\frac{\varepsilon^{1 / 2}}{q} \sqrt{\frac{\sum_{i=1}^{q-1} D V\left(x_{i}+i p / q\right)}{2 \pi}}+O(\varepsilon) .
$$

However, sometimes there are no solutions of the equation $\sum_{i=1}^{q-1} V\left(x_{i}+i p / q\right)=0$ and the eigenvalues depend on values higher than $\frac{1}{2}$ of the exponent of $\varepsilon$. This happens when the Fourier expansion of $V(x)$ has a null coefficient in the $n$-th harmonic term. In this case the amplitude of resonance depends on the other harmonics of the perturbation function and we cannot compute this amplitude of resonance in a straightforward manner using the first order approximation of our map.

\section{APPENDIX C: ALTERNATIVE METHOD TO FIND A RESONANT NORMAL FORM}

We sketch here a different procedure to determine a normal form around monotone periodic orbits of a general standard map (2-2). In this case we choose $V$ as an antisymmetric function but it is possible to carry out our computation without this assumption. Details of how to obtain these resonant normal forms are given in [Olvera and Simó 1989]. Here is an outline:

1. Given a rational rotation number $p / n$, compute an explicit form of the $n$-th iterate of $F$, defined by $\left(x_{n}, y_{n}\right)=F^{n}\left(x_{0}, y_{0}\right)$.

2. Obtain the root of this map by solving the equation $F^{n}(x, y)-(x, y)-(p, 0)=0$. The set of solutions of this equation corresponds to a monotone periodic orbit with rotation vector $p / n$.

3. Starting with a scalar transformation $y=\varepsilon \zeta_{\{0\}}+$ $p / n$ we can find a set of maps $F_{m}^{n}$, such that the difference between the map $F_{m}^{n}$ and the identity is of order $O\left(\varepsilon^{m}\right)$. For each scale transformation, $\zeta_{\{m\}}=$ $\varepsilon \zeta_{\{m+1\}}+\varphi_{\{m+1\}}(x)$ where $\zeta_{\{m+1\}}$ is the new axial coordinate, $\varphi_{\{m+1\}}(x)$ is a periodic function of the angular coordinate $x$, the new map is closer to the identity map than the previous one, the distance is of order $O\left(\varepsilon^{m+1}\right)$.

4. The lowest-order term of the new map, after $m$ transformations, is of order $O\left(\varepsilon^{m}\right)$. Consider only the lowest-order terms, which are polynomials in the axial coordinate $\zeta_{\{m\}}$ whose coefficients are periodic functions of the angular variable $x$. The fixed points of this map correspond to monotone periodic orbits of the initial map. The fixed points are obtained when $\zeta_{\{m\}}=0$; therefore the maximum number of fixed points is related to the maximum harmonic of the periodic term which depends on $x$, the number of fixed points is equal to the period of the corresponding monotone periodic orbit of the initial map. The set of monotone periodic orbits is well ordered with respect to the rotation number, and the Aubry-Mather theory shows that there exist monotone periodic orbits for any rational rotation number that belongs to the rotation interval of the twist map [Mather 1982]. If the maximum harmonic is less than $n$, where $n$ is the period of the monotone periodic orbit we want to find, it is impossible to obtain a periodic orbit with period $m$. This means that the periodic term of order $O\left(\varepsilon^{m}\right)$ which does not depend on $\zeta_{\{m\}}$ must be null otherwise we can obtain a monotone periodic orbit located in a wrong order. Therefore we can find the next scale transformation where the lower order terms are of order $m+1$. We can repeat the procedure until the maximum harmonic of the periodic terms, which depend on $x$, is equal to $n$. We denote by $S_{n}$ the number of scale transformations that we can perform until we get the maximum harmonic equal to $n$.

After performing $S_{n}$ scale transformations, we get a map that looks like a time- $\varepsilon^{S_{n}}$ flow of a Hamiltonian system. This system is a chain of $n$-pendula, the phase space of which looks like the diagram in Figure 1. The differential equation of the system is 
given in (2-9). From this equation, we can estimate the amplitude of resonance $\Delta_{p / q}$, this amplitude is given in equation $(2-10)$.

Now we show how to compute $F^{n}$. First rewrite $(2-2)$ as a second-order difference equation:

$$
x_{i+2}=2 x_{i+1}-x_{i}+\sum_{j=1}^{N} \varepsilon^{a_{j}} c_{j} \sin \left(2 \pi\left(j x_{i+1}\right)\right),
$$

where the function $V(x)$ was substituted by equation $(2-1)$, in this case we rewrite the perturbation function in terms of trigonometric series.

Using basic properties of Bessel functions it is possible to obtain the $n$-th iteration of the angular variable, $x_{n}$, in terms of the initial values $x_{0}$ and $x_{1}$. Taking this relation, we obtain the map $F^{n}$ :

$$
x_{n}=n x_{1}-(n-1) x_{0}+\sum_{i=0}^{n-2}(n-i-1) P_{i}\left(x_{0}, x_{1}\right),(\mathrm{C}-1)
$$

where

$$
\begin{array}{r}
P_{m}(x, \bar{x})=\sum_{i=1}^{N} \varepsilon^{d_{i}} q_{i} \sum_{\substack{l_{b}^{a} \in \mathbb{Z} \\
a=1, \ldots, m \\
b=1, \ldots, N}}\left(\prod_{s=1}^{N} \prod_{t=1}^{m} J_{l_{s}^{t}}\left(\varepsilon^{d_{s}} q_{s} S_{N, i}^{t}\right)\right) \\
\times \sin \left(S_{N, i}^{m+1} \bar{x}-S_{N, i}^{m} x\right),
\end{array}
$$

where $J_{i}(x)$ are the Bessel functions of integer order $i$ and the coefficients $S_{\alpha, i}^{\beta}$ are defined by:

$$
S_{\alpha, i}^{\beta}=2 \pi \sum_{r=0}^{\beta-1}(\beta-r) \sum_{j=1}^{\alpha} j l_{j}^{r}
$$

the set of numbers $l_{j}^{r}$ are integers with the restriction $l_{j}^{0}=\delta_{j}^{i}$, where $\delta_{j}^{i}$ is the Kronecker delta function.

Now, it is convenient to write the second order difference equation $(\mathrm{C}-1)$ as two difference equations, where we define $y_{i+1}=x_{i+1}-x_{i}$, for $i=0, \ldots, n$. Then we obtain

$$
\begin{aligned}
& y_{n}=y_{0}+\sum_{j=0}^{n-1} P_{j}\left(x_{0}-y_{0}, x_{0}\right), \\
& x_{n}=x_{0}+n y_{0}+\sum_{j=0}^{n-1}(n-j) P_{j}\left(x_{0}-y_{0}, x_{0}\right) .
\end{aligned}
$$

This can further be written as a power series of the perturbation parameter $\varepsilon$ :

$$
\begin{aligned}
& y_{n}=y_{0}+\sum_{j=0}^{n-1} \sum_{s \in R_{N}} \varepsilon^{s} G_{j}^{s}\left(x_{0}, y_{0}\right) \\
& x_{n}=x_{0}+n y_{0}+\sum_{j=0}^{n-1}(n-j) \sum_{s \in R_{N}} \varepsilon^{s} G_{j}^{s}\left(x_{0}, y_{0}\right),
\end{aligned}
$$

where the functions $G_{j}^{s}(x, y)$ have the definition

$$
G_{j}^{s}(x, y)=\sum_{A(j, N, i, s)} \mathcal{G}_{(j, N, i, s)} \sin \left(\bar{S}_{N, i}^{j+1} x+S_{N, i}^{j} y\right) .
$$

The coefficients $\mathcal{G}_{(j, N, i, s)}$ are constant rational numbers. The sets of index $R_{N}$ and $A(j, N, i, s)$ define the domain of the index $s, l_{a}^{b}, i$ and $t_{c}$ in the form

$$
R_{N}=\left\{s \in \mathbb{Z}: s=\sum_{t=1}^{N} w_{t} a_{t} \text { and } w_{t} \in \mathbb{Z}^{+}\right\} ;
$$

the numbers $a_{t}$ are the exponents of the perturbation parameter in equation $(2-1)$. The set $A_{(j, N, i, s)}$ defines the domain of values of the sets of integers $\left\{l_{r}^{b}\right\}$, where $l_{r}^{b} \in \mathbb{Z}, r=1, \ldots, m$ and $b=1, \ldots, N$, the set $\{i\}$ such that $i=1, \ldots, N$ and the set $\left\{t_{c}\right\}$ where $t_{c} \in \mathbb{Z}^{+}$. These sets of integers satisfy the restriction

$$
a_{i}+\left(\sum_{k=1}^{N} \sum_{b=1}^{m} a_{b}\left|l_{k}^{b}\right|\right)+\sum_{r=1}^{N} 2 t_{r} a_{r}=s .
$$

The coefficient $\bar{S}_{\alpha, i}^{\beta}$ are defined by:

$$
\bar{S}_{\alpha, i}^{\beta}=2 \pi \sum_{r=0}^{\beta-1} \sum_{j=1}^{\alpha} j l_{j}^{r} .
$$

We can check that for a fixed value of the integer $s$ the domain of index $l_{r}^{b}$ is bounded, therefore the maximum value of the coefficients $\bar{S}_{N, i}^{j+1}$ and $S_{N, i}^{j}$ are also bounded.

The map $(\mathrm{C}-2)$ can be understood as a power series in $\varepsilon$ where the coefficients are a finite Fourier series of coordinates $x$ and $y$. The maximum harmonic of any of these Fourier series corresponds to the maximum value of $\bar{S}_{N, i}^{j+1}$ and $S_{N, i}^{j}$.

\section{ACKNOWLEDGMENT}

The author is grateful to Dr. Carles Simó for helpful discussions and comments on the manuscripts and also for the computation facilities. The author is also grateful to Ana Cecilia Perez for the computer facilities in our laboratory. 


\section{REFERENCES}

[Abramowitz and Stegun 1972] M. Abramowitz and I. A. Stegun (editors), Handbook of mathematical functions with formulas, graphs, and mathematical tables, edited by M. Abramowitz and I. A. Stegun, Dover, New York, 1972. Reprinted 1992.

[Chirikov 1979] B. V. Chirikov, "A universal instability of many-dimensional oscillator systems", Phys. Rep. 52:5 (1979), 264-379.

[Escande 1982] D. F. Escande, "Renormalization for stochastic layers", Phys. D 6:1 (1982), 119-125.

[Greene 1979] J. M. Greene, "A method for determining a stochastic transition", J. of Math. Phys. 20:6 (1979), $1183-1201$.

[Lichtenberg and Lieberman 1983] A. J. Lichtenberg and M. A. Lieberman, Regular and stochastic motion, Springer, New York, 1983.
[Mather 1982] J. N. Mather, "Existence of quasiperiodic orbits for twist homeomorphisms of the annulus", Topology 21:4 (1982), 457-467.

[Olvera and Simó 1987] A. Olvera and C. Simó, "An obstruction method for the destruction of invariant curves", Phys. D 26:1-3 (1987), 181-192.

[Olvera and Simó 1989] A. Olvera and C. Simó, "Normal forms close to invariant circles in the twist map", pp. 438-443 in European Conference on Iteration Theory (ECIT 87) (Caldes de Malavella, Spain, 1987), edited by C. Alsina et al., World Scientific, Singapore, 1989.

[Simó 1990] C. Simó, "Analytical and numerical computation of invariant manifolds", pp. 285- in Modern methods in celestial mechanics (Goutelas, France, 1989), edited by D. B. et C. Froeschlé, Editions Frontières, Gif-sur-Yvette, France, 1990.

[Veerman 1993] P. Veerman, "Dynamical systems and twist maps", preprint, Centre de Reserca Matematica, Univ. Auton. de Barcelona, Bellaterra, Spain, 1993.

Arturo Olvera, IIMAS-UNAM, FENOMEC, Apdo. Postal 20-726, México D. F. 01000, Mexico (aoc@uxmym1.iimas.unam.mx)

Received April 1, 1999; accepted in revised form January 26, 2001 